Pensamiento Crítico N. ${ }^{\circ} 13$, pp. 43-55

\title{
Mercado de capitales, intermediación financiera y crecimiento económico en el Perú: 1998-2008
}

\author{
Mg. Gaby Cortez Cortez
}

\section{RESUMEN}

En concordancia con trabajos teóricos y evidencia empírica sobre la relación entre el desarrollo de los intermediarios financieros y del mercado de capitales con el crecimiento económico, se ha encontrado la existencia de una relación positiva entre el desarrollo del sistema financiero peruano con el crecimiento económico durante el periodo 1998-2008. Estos resultados sugieren que el desarrollo de los bancos medido a través del crédito bancario, apoya y sigue al crecimiento económico, y por lo tanto sugiere que los bancos solo cumplen una función estrictamente técnica de asignación de recursos en el proceso productivo.

De otro lado, la evidencia empírica nos señala que el crecimiento económico no es explicado por el desarrollo del mercado de capitales, concluyéndose que el mercado de capitales no es una fuente importante de recursos financieros para el crecimiento de la actividad económica en el Perú.

Palabras clave: Sistema financiero; mercado de capitales; crecimiento económico. 


\title{
Pensamiento Crítico N. 13
}

\begin{abstract}
In line with theoretical and empirical evidence on the relationship between development of financial intermediaries and capital markets with economic growth, we have found the existence of a positive relationship between the developments of the Peruvian financial system and economic growth during the period 19982008. These results suggest that the development of banks measured by bank credit, support and follow economic growth, and therefore suggests that banks only play a strictly technical function of allocating resources in the production process.

On the other hand, empirical evidence shows us that economic growth is not explained by capital market development, concluding that the capital market is not an important source of financial resources for the growth of economic activity in Peru.
\end{abstract}

Keywords: Financial system, capital market, economic growth.

\section{Introducción}

Durante casi dos décadas el sistema financiero peruano ha formado parte de toda una corriente de liberalización del sistema financiero mundial, expresada en una rápida expansión de los mercados de capitales en muchos países emergentes, y el ingreso de flujos financieros provenientes de economías desarrolladas. En el Perú esta corriente liberalizadora tuvo su expresión en la desregulación del mercado financiero, la privatización de empresas del Estado y el ingreso de flujos financieros de competidores extranjeros para la compra de empresas bancarias nacionales bajo diversas modalidades; así como para la adquisición de valores dentro del mercado de capitales.

La relación entre la naturaleza de la expansión de los mercados y el crecimiento económico ha motivado que un número creciente de investigadores estén interesados en establecer empíricamente relaciones de largo plazo entre: (i) el mercado de capitales, (ii) el sector bancario y (iii) el desarrollo económico (Kassimatis y Spyrous, 2000).

El presente trabajo pretende encontrar resultados sobre el rol que juegan tanto el mercado de capitales como el sector bancario en una economía liberalizada como la del Perú; mediante una visión de conjunto que permita establecer los vínculos en ambas direcciones entre la actividad económica y el sistema financiero. 


\section{Gaby Cortez Cortez}

\section{Revisión de la literatura}

Ross Levine y Sara Zervos, en su trabajo Stock Markets, Banks, and Economic Growth (1998), señalan que históricamente la relación entre el sistema financiero y el crecimiento económico se ha centrado en los bancos, tal como se argumenta en los trabajos de Walter Bagehot (1837) y Joseph Schumpeter (1912). Mientras que en contraste, Robert Lucas Jr. (1988) argumenta que los economistas han otorgado "demasiado énfasis" al rol del sistema financiero; y Joan Robinson (1952) sostiene que los bancos responden pasivamente al crecimiento económico. De manera empírica Robert King y Ross Levine (1993) muestran que el nivel de intermediación financiera sirve para hacer buenas predicciones de tasas de crecimiento económico de largo plazo, de acumulación de capital y de mejoras en la productividad.

En lo referente a la relación entre el mercado de capitales y el crecimiento económico de largo plazo, se plantean los modelos de liquidez y de riesgo. En el primero de estos modelos contrastado por Levine (1991) y Bencivenga (1995) se encuentra que los mercados de capitales más líquidos impulsan el crecimiento de la productividad. En el caso de los segundos modelos, Devereux y Smith (1994) y Obstfeld (1994) hallan que el riesgo compartido internacionalmente a través de mercados de capitales integrados, acelera el crecimiento de la productividad. En el trabajo se muestra que la liquidez del mercado de valores y el desarrollo de los bancos predicen positivamente el crecimiento, la acumulación de capital y la mejora de la productividad. Al mismo tiempo se encuentra que el tamaño del mercado de valores, la volatilidad y la integración internacional no están vinculados de manera significativa con el crecimiento.

De otro lado, el trabajo de Konstantinos Kassimatis y Spyros Spyros, Stock and credit market expansion and economic development in emerging markets: further evidence utilizing cointegration analysis (2001), sostiene que durante las dos últimas décadas los Mercados de Valores Emergentes se han expandido rápidamente, indicándose que este crecimiento se debe a la liberalización financiera y al aumento en los flujos de los portafolios extranjeros de los países industrializados. El motivo de la atracción era la baja correlación que estos mercados mostraban en relación con los mercados industrializados, así como por los beneficios potenciales de la diversificación del portafolio. Igualmente, otro encanto adicional eran las buenas perspectivas de crecimiento económico de estos mercados. También se señala que un aspecto que no ha sido estudiado de manera 


\section{Pensamiento Crítico N. 13}

adecuada es la naturaleza de la relación entre el crecimiento de los Mercados de Valores Emergentes y el desarrollo económico.

En tal sentido, los autores investigan la relación entre el desarrollo del mercado de acciones y el de crédito bancario con el desarrollo económico, para una muestra de cinco mercados de valores emergentes importantes. Los resultados parecen indicar que los mercados de acciones han jugado un rol trascendental en el caso de las economías relativamente liberalizadas, como Chile y Méjico. Mientras que en economías financieramente reprimidas, tal como en el caso de la India, el mercado de acciones no afecta el crecimiento del sector real. Además, se esbozan algunas particularidades de la naturaleza de este tipo de mercados, tal como el caso de los mercados de acciones especulativas y su relación negativa con el crecimiento económico.

Peter Rousseau y Sheng Xiao, en su trabajo "Banks, stock markets, and China's great leap forward" (2007) sostienen que China se ha convertido en la economía de crecimiento más rápido desde 1995 al 2005, habiéndose incrementado su PBI per cápita a una tasa promedio anual de 9,1\% durante estos años, y la inversión fija real per cápita aumentó a una tasa de $15 \%$ anual. Este país ha pasado de ocupar el décimo lugar al cuarto lugar en el mundo dentro de las economías más grandes, siguiendo a los Estados Unidos, Japón, y Alemania. Este crecimiento sorprendente, ha estado acompañado de reformas estructurales, dentro de las que se incluye las del sector financiero. Estos cambios se llevaron a cabo de manera importante a partir de 1995 cuando se instituyeron las leyes del Banco Central y de la Banca Comercial, a fin de establecer las bases de un sistema financiero competitivo. Con la aparición de la Bolsa de Shangai en 1990 y la Bolsa de Shenzhen en 1991, se inició un periodo de reestructuración financiera, que fue seguido por la promulgación de la Ley de Sociedades (1993) y la Ley de Valores (1998). A pesar del rápido crecimiento del mercado de valores de China, existe la duda sobre la calidad de este mercado. En este trabajo se examina el rol que han jugado los factores financieros en el reciente aumento de la actividad del sector real en China. Se describen los retos institucionales que China enfrenta para construir un mercado de capitales que funcione bien, y que complemente al sistema bancario, lo que se muestra a través de un modelo específico en donde se encuentra que el sector bancario ha sido fundamental para este éxito. 


\section{Gaby Cortez Cortez}

\section{Problema}

¿Es importante el desarrollo del mercado de capitales y de los bancos para promover el crecimiento económico de largo plazo?

¿Un mercado de capitales líquido y un sector bancario desarrollado predicen positivamente el crecimiento económico?

\section{Hipótesis}

- Hipótesis general. El desarrollo del mercado de capitales y de los bancos se encuentra positivamente relacionado con el crecimiento económico.

- Hipótesis secundaria. La liquidez del mercado de capitales y el desarrollo bancario están positivamente relacionados con el crecimiento económico.

\section{Análisis de resultados}

Crecimiento de la economía y crédito bancario

Regresión 1

En esta regresión se mide la relación entre el crecimiento de la economía con el crédito bancario, la inversión bruta fija y el ahorro interno.

Estimation Command:

$===============$

LS PROD CRED INBF AHOI

Estimation Equation:

$=============$

$\mathrm{PROD}=\mathrm{C}(1) * \mathrm{CRED}+\mathrm{C}(2) * \mathrm{INBF}+\mathrm{C}(3) * \mathrm{AHOI}$

Substituted Coefficients:

$=================$

$\mathrm{PROD}=0.2063566868 * \mathrm{CRED}+0.3325920962 * \mathrm{INBF}-0.160437161 * \mathrm{AHOI}$ 


\section{Pensamiento Crítico N. ${ }^{\circ}$ I3}

El crecimiento de la economía medido a través del crecimiento del PBI real (PROD), nos muestra que éste se encuentra explicado de manera positiva en $0,20 \%$ por el crecimiento del $1 \%$ del Crédito bancario (CRED), y en 0,33\% por el crecimiento de la Inversión Bruta Fija (INBF, proxy del stock de capital); mientras que el Ahorro financiero (AHOI) tiene una relación negativa de $0,16 \%$.

Debido al proceso de liberalización financiera introducido en la economía peruana a partir de los inicios de los años noventa, el mercado bancario peruano ha estado sujeto al mecanismo de la competencia, lo que condujo a una disminución de los márgenes bancarios, y a una mejora de la eficiencia de dichas instituciones, haciendo posible el acceso de sus servicios a un mayor número de clientes. Esto se refleja en la mejora del nivel de profundización bancaria al pasar la relación de los Depósitos/PBI de $16 \%$ durante el periodo 1990-1999 a 24\% del 2000 al 2005. Asimismo, la relación del Crédito/PBI se incrementó de $14 \%$ a $22 \%$ durante los mismo años. De aquí se colige que las instituciones bancarias han ampliado su cobertura con los consiguientes efectos en el apoyo al crecimiento.

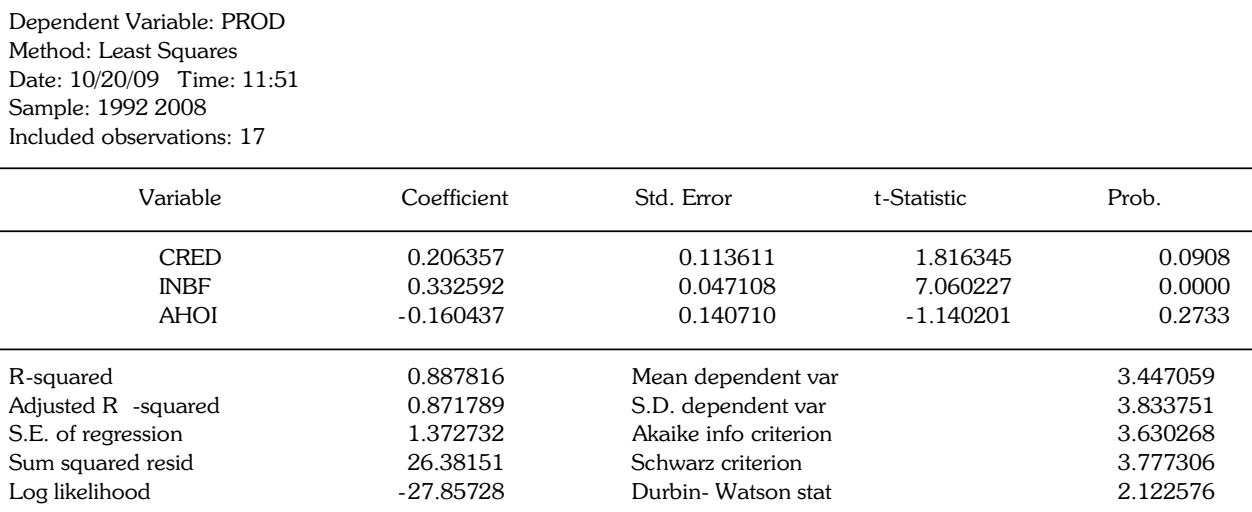

A su vez se debe tener en cuenta que el Crédito comercial y a la microempresa participó del $81,74 \%$ del monto total del crédito directo otorgado por la banca múltiple en el 2001, para luego disminuir de manera sostenida su participación hasta 68,59\% en el 2008. Mientras que el Crédito de consumo mostró una mayor significación dentro del total de crédito concedido, ya que aumenta desde 8,62\% en el 2001 hasta $18,26 \%$ en 


\section{Gaby Cortez Cortez}

el 2007. En el caso del crédito hipotecario, éste se incrementa desde 9,6\% del total de créditos otorgados en el 2001 hasta 13,6\% en el 2009, pasando por su punto más alto en el 2005 con $14,8 \%$ del total.

Se puede resumir entonces que el crédito bancario dirigido expresamente a financiar a las empresas fue el rubro más importante del negocio bancario durante el periodo de análisis, pero tuvo una pérdida de participación frente al crédito de consumo e hipotecario, lo que nos lleva a sostener que el crédito bancario solamente apoya y sigue al crecimiento económico, pero no lo determina.

La participación del Ahorro Interno medido a través del ahorro financiero tiene un signo negativo que se explica porque al crecer el producto crece el consumo, que es atendido por la banca a través de tarjetas de crédito, y por créditos hipotecarios, disminuyendo la incidencia de los créditos comerciales dirigidos a los sectores productivos, que son los que contribuyen a generar valor agregado. Además, debe señalarse que los créditos comerciales disminuyeron su participación dentro del total de créditos de la banca al pasar de $81,7 \%$ en el 2001 a $68,2 \%$ en el 2008. Unida a esta disminución está la reducción del crédito a la industria manufacturera a lo largo del periodo de análisis.

\section{Regresión 2}

En esta regresión se mide la relación entre el crédito bancario el crecimiento económico, la inversión bruta fija y el ahorro interno.

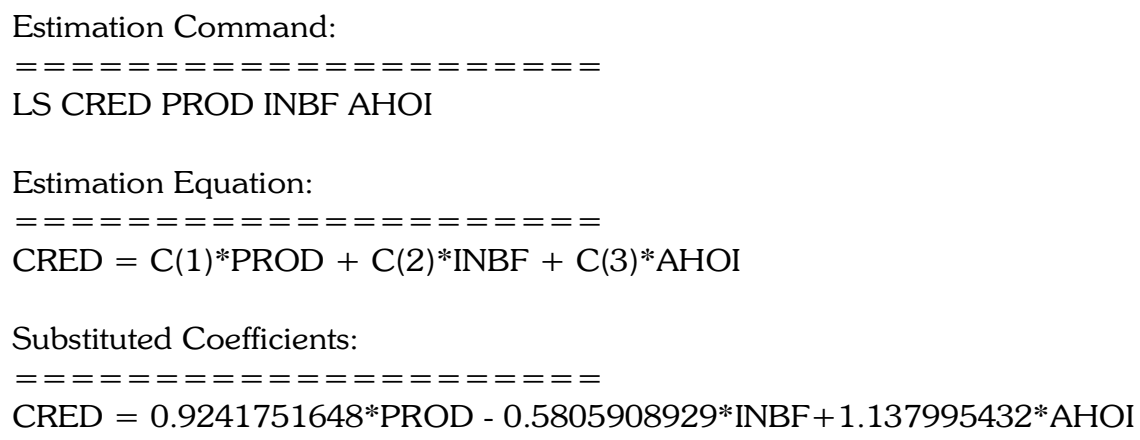

Estimation Command:

$=============$

LS CRED PROD INBF AHOI

Estimation Equation:

$=============$

$\mathrm{CRED}=\mathrm{C}(1) * \mathrm{PROD}+\mathrm{C}(2) * \mathrm{INBF}+\mathrm{C}(3) * \mathrm{AHOI}$

Substituted Coefficients:

$==============$

CRED $=0.9241751648 *$ PROD $-0.5805908929 *$ INBF $+1.137995432 *$ AHOI 


\section{Pensamiento Crítico N. I3}

En esta regresión se intercambia las variables para probar la fortaleza de las relaciones entre ellas, y se encuentra que el crecimiento del crédito se encuentra explicado en $0,92 \%$ ante el crecimiento de $1 \%$ del PBI real.

\begin{tabular}{|c|c|c|c|c|}
\hline \multicolumn{5}{|l|}{$\begin{array}{l}\text { Dependent Variable: CRED } \\
\text { Method: Least Squares } \\
\text { Date: 10/20/09 Time: } 11: 39 \\
\text { Sample: } 19922008 \\
\text { Included observations: } 17\end{array}$} \\
\hline Variable & Coefficient & Std. Error & t-Statistic & Prob. \\
\hline PROD & 0.924175 & 0.508810 & 1.816345 & 0.0908 \\
\hline INBF & -0.580591 & 0.145763 & -3.983103 & 0.0014 \\
\hline $\mathrm{AHOI}$ & 1.137995 & 0.066355 & 17.15002 & 0.0000 \\
\hline R-squared & 0.779134 & Mean dependent var & & 18.94706 \\
\hline Adjusted R -squared & 0.747582 & S.D. dependent var & & 5.782205 \\
\hline S.E. of regression & 2.905051 & Akaike info criterion & & 5.129564 \\
\hline Sum squared resid & 118.1505 & Schwarz criterion & & 5.276601 \\
\hline Log likelihood & -40.60129 & Durbin -Watson stat & & 1.803855 \\
\hline
\end{tabular}

\section{Crecimiento de la economía y Mercado de capitales}

\section{Regresión 3}

En esta regresión se mide la relación entre el crecimiento económico, la capitalización bursátil, la inversión bruta fija y el ahorro interno.

Estimation Command:

$============$

LS PROD CAPB INBF AHOI

Estimation Equation:

$=\mathrm{=}=\mathrm{=}=\mathrm{=}=\mathrm{=}=\mathrm{=}=\mathrm{=}=$

$\mathrm{PROD}=\mathrm{C}(1) * \mathrm{CAPB}+\mathrm{C}(2) * \mathrm{INBF}+\mathrm{C}(3) * \mathrm{AHOI}$

Substituted Coefficients:

$============$

$\mathrm{PROD}=0.01446716605 * \mathrm{CAPB}+0.2329939853 * \mathrm{INBF}+0.07013146125 * \mathrm{AHOI}$ 


\section{Gaby Cortez Cortez}

Se encuentra que el crecimiento de la economía se haya explicado en tan solo $0,01 \%$ por el aumento de $1 \%$ de la capitalización bursátil (CAPB), en 0,23\% por la inversión bruta fija (INBF), y en $0,07 \%$ por el ahorro interno (AHOI). Los resultados muestran que el crecimiento económico no está explicado por el movimiento del mercado de capitales.

Esta escasa influencia de la capitalización se produce a pesar de que la capitalización del mercado/PBI fue en promedio del orden de 62,9\% durante los años 20052008. Asimismo, se debe mencionar que la capitalización bursátil muestra aumentos significativos de $150 \%$ en el 2006 , de $80,3 \%$ en el 2007 ; para luego disminuir a $47 \%$ en el 2008 como resultado de la crisis financiera internacional.

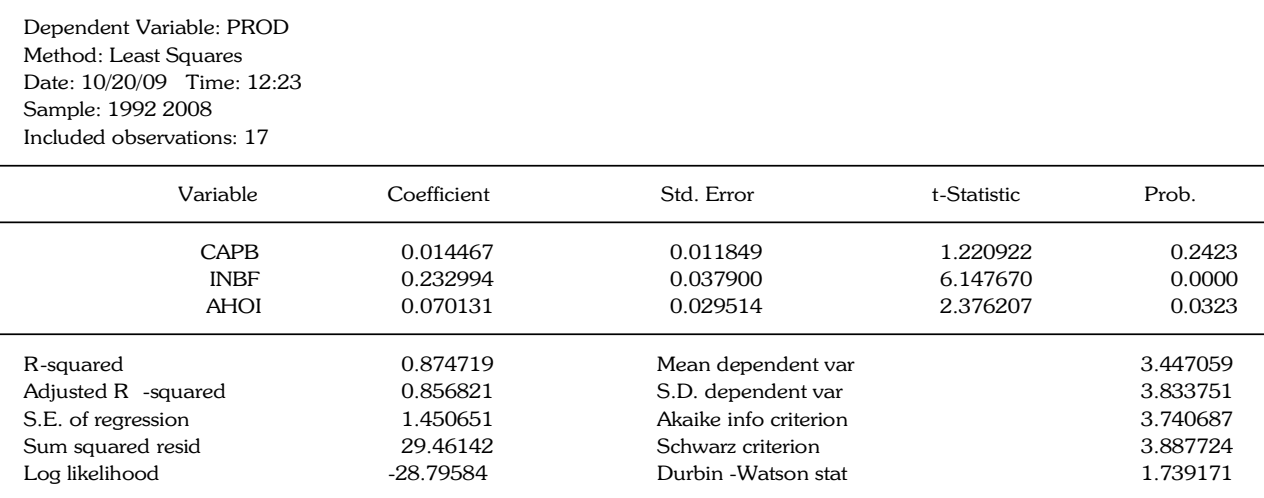

Debe señalarse que dentro del total de capitalización bursátil, las acciones mineras significaron el 57,6\% en el 2006, el 56,3\% en el 2007, y el 53\% en el 2008, indicándonos la importancia de estas acciones en el total capitalizado. Mientras que las acciones industriales pasaron de participar del $22 \%$ del total de capitalización bursátil en el 2001 a $7 \%$ en el 2008. Estos hechos nos muestran que más de la mitad de la capitalización bursátil se encuentra explicada por las acciones mineras, las que se suscriben a una actividad netamente extractiva sin valor agregado, lo que revela de cierta forma la escasa influencia del mercado de capitales en el crecimiento económico. Asimismo, se encuentra que las acciones industriales que son las que generan valor agregado, se han visto disminuidas de manera significativa durante el periodo de análisis. 


\section{Pensamiento Crítico N. I3}

\section{Regresión 4}

En esta regresión se encuentra la relación entre la capitalización bursátil, el crecimiento económico, la inversión bruta fija y el ahorro interno. En ésta se intercambia las variables para probar la fortaleza de las relaciones entre ellas,

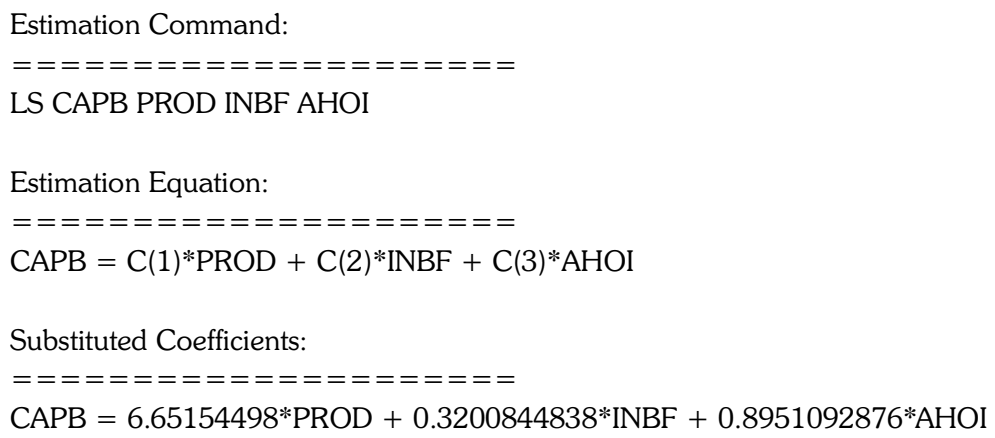

El crecimiento de la capitalización bursátil se encuentra explicado en $6,65 \%$ ante un crecimiento de $1 \%$ de la producción. El bajo coeficiente del R2 nos señala que la estimación no predice los valores de la variable dependiente de la muestra. Debe tenerse en cuenta que el 52\% de la capitalización está compuesta de acciones mineras, vinculadas a cotizaciones internacionales, que responden a movimientos de economías externas.

\begin{tabular}{|c|c|c|c|c|}
\hline \multicolumn{5}{|c|}{$\begin{array}{l}\text { Dependent Variable: CAPB } \\
\text { Method: Least Squares } \\
\text { Date: 10/20/09 Time: 12:21 } \\
\text { Sample: } 19922008 \\
\text { Included observations: } 17\end{array}$} \\
\hline Variable & Coefficient & Std. Error & t-Statistic & Prob. \\
\hline PROD & 6.651545 & 5.44797 & 1.220922 & 0.2423 \\
\hline INBF & 0.320084 & 1.560728 & 0.205087 & 0.8405 \\
\hline AHOI & 0.895109 & 0.710484 & 1.259858 & 0.2283 \\
\hline R-squared & 0.512978 & Mean depen & & 41.46471 \\
\hline Adjusted R-squared & 0.443403 & S.D. depend & & 41.69289 \\
\hline S.E. of regression & 31.10516 & Akaike info & & 9.871409 \\
\hline Sum squared resid & 13545.43 & Schwarz crit & & 10.01845 \\
\hline Log likelihood & -80.90698 & Durbin -Wat & & 2.866881 \\
\hline
\end{tabular}




\section{Gaby Cortez Cortez}

\section{Crecimiento de la economía y el Mercado de capitales a través de la Negociación Bursátil}

\section{Regresión 5}

En esta regresión se relaciona el crecimiento económico con el volumen negociado, la inversión bruta fija y el ahorro interno.

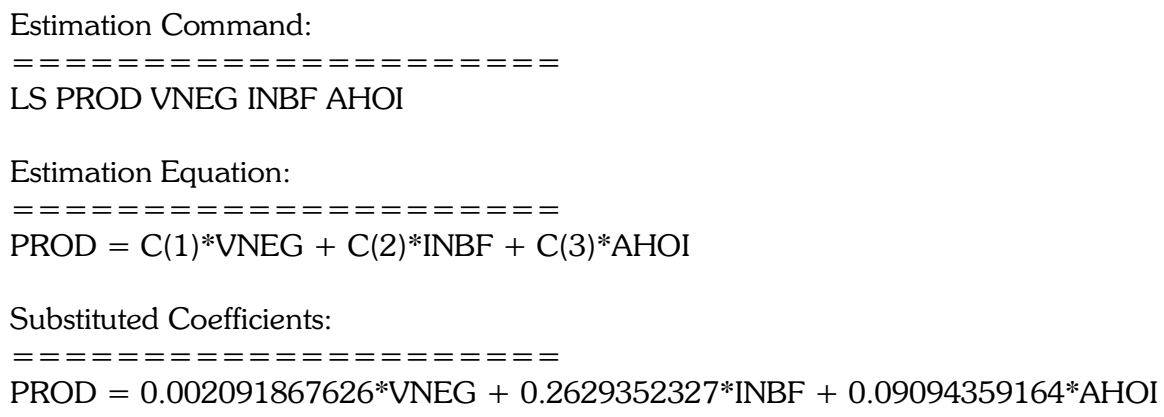

El crecimiento económico se encuentra explicado en tan solo $0,002 \%$ por un aumento de $1 \%$ en el Volumen Negociado en la BVL. El volumen negociado estadísticamente no tiene una incidencia importante en la explicación del crecimiento de la economía, debido a los valores mínimos de la negociación, señalándonos que la BVL no es un mercado de capitales líquido.

Esta escasa vinculación entre el volumen negociado en la BVL y el crecimiento económico puede verse explicado porque los montos negociados a lo largo del periodo solo muestran un comportamiento ascendente importante del 2006 al 2007, mientras que, el resto de años tiene variaciones negativas. De los datos sobre cotizaciones de acciones se observa que en varios casos no existe movimiento de precios durante semanas, ni tampoco negociación de las mismas, lo cual indica que es un mercado poco líquido.

Dentro de esta escasa negociación de valores a través de la BVL, destacan las acciones mineras comunes, que participan del 22,5 del total negociado del 2001 al 2008 , siguiéndole las acciones comunes industriales con un promedio de participación de $19,1 \%$ en el mismo periodo. 


\section{Pensamiento Crítico N. 13}

Dependent Variable: PROD

Method: Least Squares

Date: 10/20/09 Time: 16:00

Sample: 19922008

Included observations: 17

\begin{tabular}{|lllll|}
\hline Variable & Coefficient & Std. Error & t-Statistic & Prob. \\
\hline VNEG & 0.002092 & 0.080633 & 0.025943 & 0.9797 \\
INBF & 0.262935 & 0.030404 & 8.648122 & 0.0000 \\
AHOI & 0.090944 & 0.045263 & 2.009234 & 0.0642 \\
\hline R-squared & 0.861386 & Mean dependent var & 3.447059 \\
Adjusted R-squared & 0.841584 & S.D. dependent var & 3.833751 \\
S.E. of regression & 1.525890 & Akaike info criterion & 3.841818 \\
Sum squared resid & 32.59676 & Schwarz criterion & 3.988856 \\
Log likelihood & -29.65545 & Durbin-Watson stat & 1.692925 \\
\hline
\end{tabular}

\section{Conclusiones}

1. Estos resultados nos señalan la vinculación entre el crecimiento de la economía y el crédito bancario, sugiriendo que el crédito apoya y sigue al crecimiento económico y no el crecimiento económico al crédito; cumpliendo los bancos por lo tanto, una función estrictamente técnica de asignación de recursos en el proceso productivo.

2. El crecimiento económico no es explicado por el crecimiento del mercado de capitales a través de la capitalización bursátil y la negociación bursátil, ceteris paribus.

3. Se concluye que el mercado de capitales no es una fuente importante de recursos para el crecimiento económico, mientras que los intermediarios bancarios, continúan liderando el financiamiento de la economía peruana; pero jugando un papel de seguimiento de la producción, más no de impulsor del crecimiento económico.

\section{Bibliografía}

Bencivenga, Valerie; Smith, Bruce (1991). "Financial intermediation and endogenous growth".

Castro, Juan Francisco; Rivas Llosa, Roddy (2003). Econometría aplicada. Universidad del Pacífico. Centro de Investigación. 


\section{Gaby Cortez Cortez}

Greene, William (1999). Análisis econométrico. Tercera edición, Prentice Hall.

Kassimatis, Konstantinos; Spyros, Spyros (2001). Stock and credit market expansion and economic development in emerging markets: further evidence utilizing cointegration analysis.

Levine, Ross; Zervos, Sara (1998). Stock markets, banks, and economic growth.

Rousseau, Peter; Xiao, Sheng; Banks (2007). Stock markets, and China's great leap forward. 